(C) 1998 Springer-Verlag New York Inc.

\title{
Erratum \\ Continuity Properties and Global Attractors of Generalized Semiflows and the Navier-Stokes Equations
}

J. M. Ball

Mathematical Institute, University of Oxford, 24-29 St. Giles, Oxford OX1 3LB, UK e-mail: ball@maths.ox.ac.uk

Received January 5, 1998

The proof of Proposition 7.4 in [1] needs modification. The statement after (7.17) that $V\left(u^{(j)}\right)(s) \rightarrow V(u)(s)$ for a.e. $s>0$ is not obvious, and perhaps not true. However, it still follows that $u$ is a weak solution and then the subsequent argument can be applied without any essential change to the function

$$
\tilde{V}(u)(t):=\frac{1}{2}\|u(t)\|^{2}-\int_{0}^{t}(f, u(\tau)) d \tau,
$$

noting that $\tilde{V}\left(u^{(j)}\right)(s) \rightarrow \tilde{V}(u)(s)$ for a.e. $s>0$ and that $\tilde{V}\left(u^{(j)}\right)(t)$ and $\tilde{V}(u)(t)$ are nonincreasing. A similar change should be made to the last paragraph of the proof of Corollary 7.5 .

I am grateful to Gilles Francfort for querying this point.

\section{References}

[1] J. M. Ball. Continuity properties and attractors of generalized semiflows and the Navier-Stokes equations. J. Nonlinear Science, 7:475-502, 1997. 